\title{
Fine-scale spatial genetic structure of an endangered marsh herb, Caldesia grandis (Alismataceae)
}

\author{
X.-L. Yue ${ }^{1 *}$, J.-M. Chen ${ }^{2,3 *}$, Y.-H. Guo ${ }^{1}$ and Q.-F. Wang ${ }^{2,3}$ \\ ${ }^{1}$ Laboratory of Plant Systematics and Evolutionary Biology, \\ College of Life Sciences, Wuhan University, Wuhan, Hubei, China \\ ${ }^{2}$ Key Laboratory of Aquatic Botany and Watershed Ecology, \\ Chinese Academy of Sciences, Wuhan, Hubei, China \\ ${ }^{3}$ Wuhan Botanical Garden, Chinese Academy of Sciences, \\ Wuhan, Hubei, China \\ *These authors contributed equally to this study. \\ Corresponding author: Q.-F. Wang \\ E-mail: qfwang@wbgcas.cn
}

Genet. Mol. Res. 11 (3): 2412-2421 (2012)

Received September 15, 2011

Accepted January 24, 2012

Published May 18, 2012

DOI http://dx.doi.org/10.4238/2012.May.18.10

\begin{abstract}
The endangered marsh herb, Caldesia grandis, is native to China. We investigated the spatial structure of the genetic variation of three populations of $C$. grandis using RAPD markers and spatial autocorrelation analysis, based on the method of equal distance interval. A total of 157 individuals were sampled from four patches collected from the region of Hunan and Yunnan Provinces, China. Among the polymorphic bands generated by seven selective primers, polymorphic bands with frequencies ranging from 20 to $80 \%$ were used to calculate Moran's I spatial autocorrelation coefficient for each patch. We found significant spatial structure of genetic variation in the three patches in Bei Hai (BH) (patches BH-1 and BH-2) and Guai Hu (GH) (patch GH1) populations of $C$. grandis (with significant positive autocorrelation within the short distance class). In contrast, the genetic variation in
\end{abstract}


the Lang Pan $\mathrm{Hu}(\mathrm{LPH})$ population (patch LPH-1) was found to be randomly distributed. The different spatial distribution patterns may be attributed to environment differences. These results have implications for the conservation and management of this species, especially for sampling strategies for ex situ conservation.

Key words: Caldesia grandis; Conservation genetics; Endangered species; Spatial genetic structure

\section{INTRODUCTION}

Abundant evidence has demonstrated that plant populations could exhibit spatial genetic structures even though random or near random distribution have been found in some cases (Escudero et al., 2003). Genetic diversity may appear spatially structured on different scales, such as medium and large scales (population or region; Hedrick, 1986; Heywood, 1991; Born et al., 2008) or among neighboring individuals (Sokal et al., 1989; Epperson, 1993; Li et al., 2002; Reisch et al., 2007). Even at extremely fine scales, spatial genetic structures have been detected in some plant populations (Epperson and Clegg, 1986; Tani et al., 1998; He et al., 1999, 2000; Torres et al., 2003; Vornam et al., 2004). Spatial genetic structure within plant populations is primarily determined by the effects of factors such as limited seed and pollen dispersal, vegetative reproduction, isolation in small patches, differential mortality, and microhabitat selection (Murawski and Hamrick, 1990; Epperson, 1993; Berg and Hamrick, 1994; Chung and Epperson, 1999). Analysis of genetic diversity in a spatial context can provide new insight in the understanding of the mechanisms of population maintenance and dynamics; thus, knowledge of the spatial structure of populations is important when selecting natural populations for conservation or sampling them for breeding programs (Heywood, 1991; He et al., 1999; Escudero et al., 2003).

Caldesia grandis Samuelsson is an erect, perennial marsh herb that belongs to the aquatic family Alismataceae. C. grandis is a self-compatible species that can reproduce both sexually with selfed and out-crossed seeds and vegetatively through bulbils that commonly occur in the inflorescences (Gituru et al., 2002). C. grandis produces many flowers and seeds, but seedling recruitment is rarely observed (Gituru et al., 2002). This species is confined to mountainous bogs and marshes in Southeast Asia and has been found in China and the eastern Himalayas (Cook, 1996). C. grandis in China has been reported in Hubei, Hunan, Guangdong, and Yunnan Provinces in mainland China as well as on the island of Taiwan (Gituru et al., 2002). The species is rare, occurring as small populations in China. In our recent field investigation, only three natural populations including one population in Yunnan Province and two populations in Hunan Province were found in mainland China (Chen et al., 2006).

The development of appropriate strategies for the conservation and exploitation of plant genetic resources requires a detailed knowledge of not only the amount but also the spatial distribution of genetic variation within a species (Escudero et al., 2003). In a previous research, Chen et al. (2006) studied the extent of clonality and amount of genetic diversity in the three known populations of $C$. grandis in mainland China. They found a high level of intra-population and a low level of inter-population genetic variation in this endangered marsh herb and proposed that sexual reproduction may have played an important role at some 
point during the history of these populations. To link knowledge of genetic diversity to certain ecological traits of the species and to obtain a better understanding of the biological process that govern the dynamics of the populations, we explored the existence of nonrandom spatial genetic structures within the $C$. grandis populations.

The random amplified polymorphic DNA (RAPD) technique has several advantages over isozyme and other DNA marker methodologies. These advantages include speed, low cost, and the use of small amounts of plant material (Huff et al., 1993; Heun et al., 1994; Fritsch and Rieseberg, 1992). In other studies, the spatial genetic structure of several plant species have been successfully investigated using RAPD markers (Bucci and Menozzi, 1995; Degen et al., 2001; Bouza et al., 2002; Torres et al., 2003). In a study of the population genetics of $C$. grandis, two molecular marker systems, RAPD and inter-simple sequence repeat, were used (Chen et al., 2006). Both of the markers worked effectively in clone identification of C. grandis. RAPD markers detected more diversity than inter-simple sequence repeat markers in the three populations examined, however (Chen et al., 2006). In the present study, we used RAPD molecular markers to assess the fine-scale spatial genetic structure within three populations of C. grandis. The information on spatial genetic variation gained in this study may facilitate the conservation management of this species.

\section{MATERIAL AND METHODS}

\section{Study sites and sampling}

We examined three wild populations of $C$. grandis occurring in three marshes, Lang Pan Hu, Guai Hu, and Bei Hai (referred to as the LPH, GH, and BH populations, respectively), in the Hunan and Yunnan Provinces of China. In Hunan Province, C. grandis is found in two populations ( $\mathrm{LPH}$ and $\mathrm{GH}$ ) close to the center of the Mangshan Nature Reserve. The LPH marsh $\left(24^{\circ} 52^{\prime} \mathrm{N} ; 112^{\circ} 43^{\prime} \mathrm{E}\right)$ is densely covered with a thick mat composed mainly of the peat moss Sphagnum cuspidatum and the fern Cyclosorus acuminatus. A sluggish stream flows across the marsh, and the marsh is subdivided by patches of the bamboo Sinarundinaria nitida. The GH marsh $\left(25^{\circ} 03^{\prime} \mathrm{N} ; 113^{\circ} 00^{\prime} \mathrm{E}\right)$ is approximately $8 \mathrm{~km}$ from the $\mathrm{LPH}$ marsh and is subdivided by patches of a canopy, Brasenia schreberi. C. grandis in the $\mathrm{BH}$ marsh $\left(25^{\circ} 07^{\prime} \mathrm{N}\right.$; $\left.98^{\circ} 33^{\prime} \mathrm{E}\right)$ population is found in four patches in Yunnan Province. Leaves of plants from the LPH population were collected from one of the patches (LPH-1). Leaves of plants from the GH population were also collected from one patch (GH-1); those of the $\mathrm{BH}$ population were collected from two patches (BH-1 and BH-2). We sampled all of the individuals in each patch. A total of 157 individuals were sampled: 64 from LPH-1, 38 from GH-1, 31 from BH-1, and 24 from BH-2. The locations of the sampled plants are shown in Figure 1. Each individual within each of the four patches was characterized by its location on the grid ( $\mathrm{x}, \mathrm{y}$ coordinates).

\section{DNA extraction and RAPD amplification}

Total genomic DNA was isolated from $0.5 \mathrm{~g}$ silica-dried leaf tissue following a procedure described by Chen et al. (2006). RAPD reactions were carried out in a volume of 25 $\mu \mathrm{L}$ containing $0.25 \mathrm{mM}$ of each dNTP, $2.5 \mu \mathrm{L}$ 10X Taq buffer (10 mM Tris-HCl, PH 8.3, 1.5 $\mathrm{mM} \mathrm{MgCl}_{2}$ and $50 \mathrm{mM} \mathrm{KCl}$ ), $1 \mathrm{mM}$ primer, $1 \mathrm{U}$ Taq Polymerase (Tian Yuan Biotech, Wuhan, 
China), and 40 ng DNA template. Amplification of genomic DNA was carried out on a PTC$100^{\mathrm{TM}}$ thermocycler (MJ Research Inc.) and commenced with 4 min at $94^{\circ} \mathrm{C}$ followed by 45 cycles of $1 \mathrm{~min}$ at $94^{\circ} \mathrm{C}, 1 \mathrm{~min}$ at $34^{\circ} \mathrm{C}$, and $2 \mathrm{~min}$ at $72^{\circ} \mathrm{C}$, and ended with $7 \mathrm{~min}$ at $72^{\circ} \mathrm{C}$. Amplification products were resolved electrophoretically on $1.5 \%$ agarose gels run at $100 \mathrm{~V}$ in $0.5 \mathrm{X}$ tris-boric acid-ethylenediaminetetraacetic acid, visualized by staining with ethidium bromide, and photographed under ultraviolet light. Sixty RAPD primers from Genbase Co. Ltd. (Shanghai, China) were screened on eight randomly selected individuals. The eight samples were amplified twice with the same primer. Seven primers that produced clear and $100 \%$ reproducible fragments were selected for further analysis (Table 1).

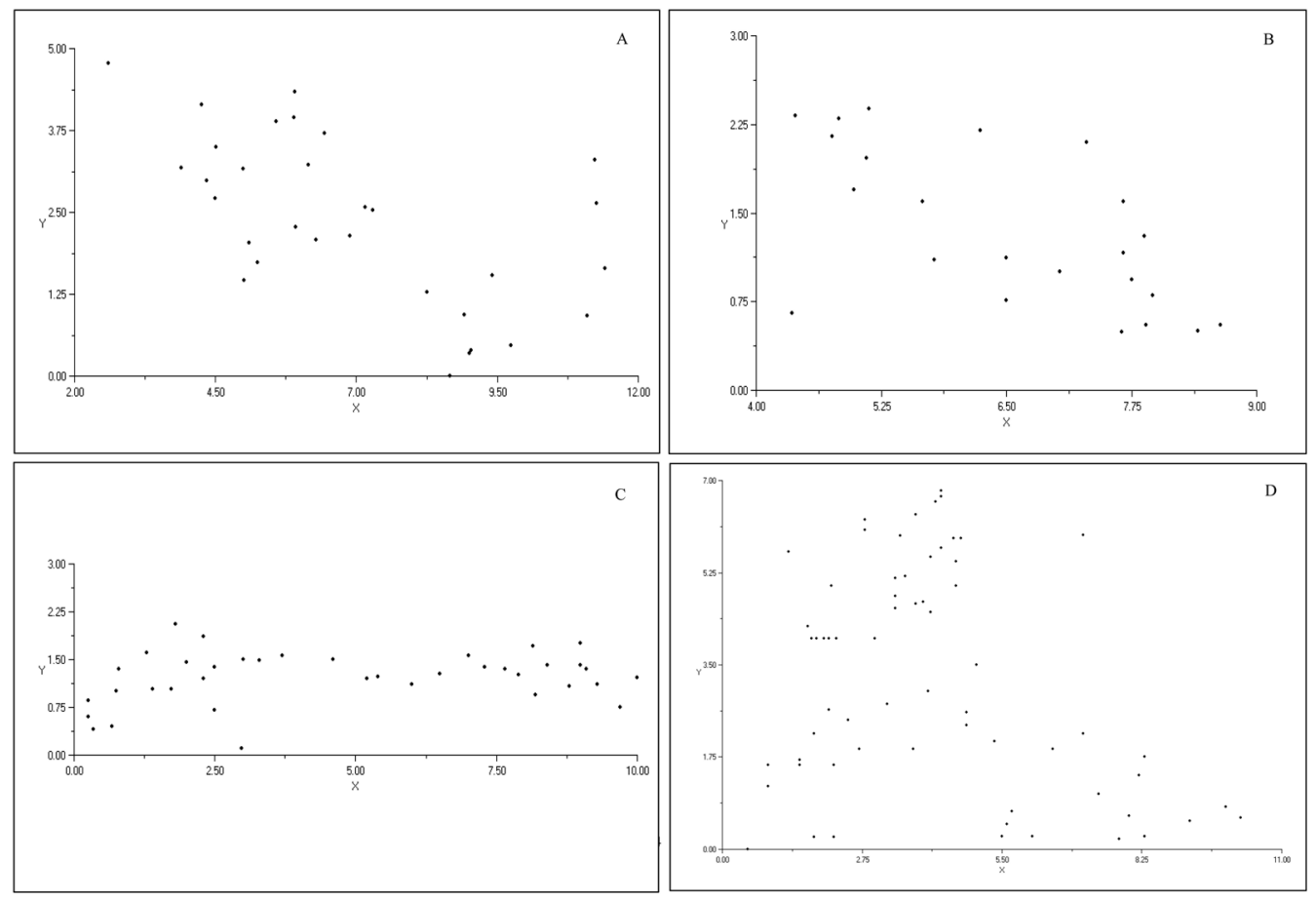

Figure 1. Spatial distribution of Caldesia grandis individuals. A. Patch BH-1 of the Bei Hai Marsh (BH) population; B. Patch BH-2 of BH population; C. Patch GH-1 of Guai Hu Marsh population; D. Patch LPH-1 of the Lang Pan Hu Marsh population.

Table 1. Number of bands scored from RAPD primers.

\begin{tabular}{lcc}
\hline Primers & Number of scorable bands & Number of polymorphic bands \\
\hline P-A13: 5'-CCTGGGTGGA-3' & 8 & 8 \\
P-A14: 5'-CCTGGGTTC-3' & 9 & 7 \\
P-A19: 5'-GCCCGGTTTA-3' & 11 & 10 \\
P-B03: 5'-CTCCCTGAGC-3' & 7 & 4 \\
P-B08: 5'-TTCCCGGAGC-3' & 10 & 9 \\
P-B09: 5'-TTCCGGGTGC-3' & 8 & 8 \\
P-C15: 5'-TTCCGCGGGC-3' & 8 & 6 \\
\hline
\end{tabular}




\section{Data analysis}

RAPD amplification products were treated as phenotypes and each band was scored as present (1) or absent (0) for each sample. Thus, a matrix of RAPD phenotypes was formed for each patch in each population. The testing for the presence of spatial autocorrelation within each patch was approached with Moran's I correlogram (Sokal and Oden, 1978a) using the SAAP 4.3 program written by Warternberg (1989). In spatial autocorrelation analysis, when the sample size is large, the expected Moran's $I$ value $\left(E_{(I)}\right)\left(E_{(I)}=-1 /(n-1)\right.$, where $n=$ sampled site) is close to 0 . An observed Moran's $I$ value significantly greater than the expected value indicates similarity between two sampled sites; dissimilarity between two sampled sites is indicted by the opposite (Sokal and Oden, 1978a,b).

In spatial autocorrelation analysis, if the frequencies of the polymorphic loci are too high or too low, they provide only limited information. To avoid unnecessary calculations while ensuring that the data has sufficient statistical power in the spatial autocorrelation analysis, we used only the polymorphic bands with frequencies ranging from 20 to $80 \%$ generated by the selective primers to calculate Moran's I spatial autocorrelation coefficient for each patch. These analyses were conducted with an approach of equal distance interval (10 distance classes). The distance classes were set using the average distance between the individuals. Moran's I statistic was calculated for each distance class. Each value of Moran's I was tested for significant deviations from the expected value under the null hypothesis of random spatial distribution (Cliff and Ord, 1981). For patch BH2, only five distance classes were set in the analysis.

\section{RESULTS}

Of the 60 RAPD primers screened, seven produced highly reproducible bands. Using these primers, a total of 52 polymorphic loci were generated among the 157 individuals from the four patches. For patch BH-1, four loci with frequencies ranging from 20 to $80 \%$ were selected, and the average distance between individuals was $1 \mathrm{~m}$. Among the 40 calculated Moran's I values, 19 (48\%) deviated significantly from the expected value under the null hypothesis of random spatial distribution at the 5\% level based on 1000 permutations (Table 2). At the first seven of the 10 distance classes, $39 \%$ of the Moran's $I$ values were statistically significant. Within a short distance $(3 \mathrm{~m}), 100 \%$ of the statistical significant Moran's I values were positive. With the distance increased, the positive Moran's $I$ values decreased and the negative values increased. Within the distance classes of 8 to 10 , most of the Moran's I values were significantly negative at the $5 \%$ level (see Table 2; Figure 2A).

\begin{tabular}{|c|c|c|c|c|c|c|c|c|c|c|c|}
\hline \multirow[t]{3}{*}{ Locus } & \multicolumn{10}{|c|}{ Distance class $(\mathrm{m})$ and No. of individual pairs } & \multirow[t]{3}{*}{ Cumulative probability } \\
\hline & 1 & 2 & 3 & 4 & 5 & 6 & 7 & 8 & 9 & 10 & \\
\hline & 42 & 84 & 94 & 59 & 56 & 60 & 37 & 20 & 9 & 4 & \\
\hline 1 & 0.01 & 0.02 & 0.04 & -0.17 & -0.24 & -0.04 & 0.00 & 0.22 & -0.27 & 0.63 & 0.517 \\
\hline 2 & 0.14 & -0.06 & -0.10 & -0.07 & $-0.25^{*}$ & $0.24 *$ & $0.26^{*}$ & $-0.52 * *$ & 0.14 & $-1.00 *$ & 0.087 \\
\hline 3 & $0.26^{*}$ & $0.13^{*}$ & $0.20^{* *}$ & 0.06 & -0.01 & $-0.41 * *$ & -0.28 & $-0.72 * *$ & $-0.69^{*}$ & $-1.00^{*}$ & 0.004 \\
\hline 4 & $0.26^{*}$ & $0.13^{*}$ & $0.20 * *$ & 0.06 & -0.01 & $-0.41 * *$ & -0.28 & $-0.72 * *$ & $-0.69^{*}$ & $-1.00 *$ & 0.004 \\
\hline Average & 0.17 & 0.05 & 0.09 & -0.03 & -0.13 & -0.16 & -0.07 & -0.43 & -0.38 & -0.59 & \\
\hline Maximum & 0.26 & 0.13 & 0.20 & 0.06 & -0.01 & 0.24 & 0.26 & 0.22 & 0.14 & 0.63 & \\
\hline Minimum & 0.01 & -0.06 & -0.10 & -0.17 & -0.25 & -0.41 & -0.28 & -0.72 & -0.72 & -1.00 & \\
\hline
\end{tabular}


For patch $\mathrm{BH}-2,13$ loci with frequencies ranging from 20 to $80 \%$ were selected, and the average distance between individuals was $1 \mathrm{~m}$. Among the 65 calculated Moran's $I$ values, $45(69 \%)$ were statistically significant at the $5 \%$ level (Table 3). At the first four of the five distance classes, more than $50 \%$ of the Moran's $I$ values were statistically significant. Within a short distance $(2 \mathrm{~m}), 100 \%$ of the statistically significant Moran's $I$ values that were positive. Within the distance range of $4-5 \mathrm{~m}, 100 \%$ of the statistically significant Moran's $I$ values were negative at the $5 \%$ level (see Table 3; Figure 2B).

Table 3. Spatial autocorrelation coefficients (Moran's $I$ ) for 5 distance classes in patch BH-2 in Bei Hai population of Caldesia grandis (equal interval correlograms).

\begin{tabular}{|c|c|c|c|c|c|c|}
\hline \multirow[t]{2}{*}{ Locus } & \multicolumn{5}{|c|}{ Distance class (m) } & \multirow[t]{2}{*}{ Cumulative probability } \\
\hline & 1 & 2 & 3 & 4 & 5 & \\
\hline 1 & $0.31 * *$ & $0.15^{*}$ & -0.15 & $-0.40 * *$ & -0.12 & 0.005 \\
\hline 2 & $0.31 * *$ & $0.15^{*}$ & -0.15 & $-0.40 * *$ & -0.12 & 0.005 \\
\hline 3 & $0.75 * *$ & $0.47 * *$ & -0.21 & $-0.70 * *$ & $-0.52 * *$ & 0.000 \\
\hline 4 & $0.75^{* *}$ & $0.47 * *$ & -0.21 & $-0.70 * *$ & $-0.52 * *$ & 0.000 \\
\hline 5 & $0.80 * *$ & $0.23 * *$ & -0.07 & $-0.32 *$ & $-0.84 * *$ & 0.000 \\
\hline 6 & $0.66 * *$ & $0.35 * *$ & -0.06 & $-0.41 * *$ & $-0.74 * *$ & 0.000 \\
\hline 7 & $0.53 * *$ & $0.37 * *$ & -0.16 & $-0.59 * *$ & $-0.37 * *$ & 0.000 \\
\hline 8 & $0.66 * *$ & $0.35 * *$ & -0.06 & $-0.41 * *$ & $-0.74 * *$ & 0.000 \\
\hline 9 & $0.75 * *$ & $0.47^{* *}$ & -0.21 & $0.70 * *$ & $-0.52 * *$ & 0.000 \\
\hline 10 & $0.16^{*}$ & -0.21 & -0.21 & 0.03 & 0.02 & 0.02 \\
\hline 11 & $0.80 * *$ & $0.23 * *$ & -0.07 & $-0.32 *$ & $-0.84 * *$ & $-0.84 * *$ \\
\hline 12 & $0.80 * *$ & $0.23 * *$ & -0.07 & $-0.32 *$ & $-0.84 * *$ & $-0.84 * *$ \\
\hline 13 & $0.33 * *$ & 0.04 & -0.17 & $-0.45^{* *}$ & 0.03 & 0.03 \\
\hline Average & 0.59 & 0.25 & -0.14 & -0.44 & -0.47 & \\
\hline Maximum & 0.80 & 0.47 & -0.06 & 0.03 & 0.03 & \\
\hline Minimum & 0.16 & -0.21 & -0.21 & -0.70 & -0.84 & \\
\hline
\end{tabular}
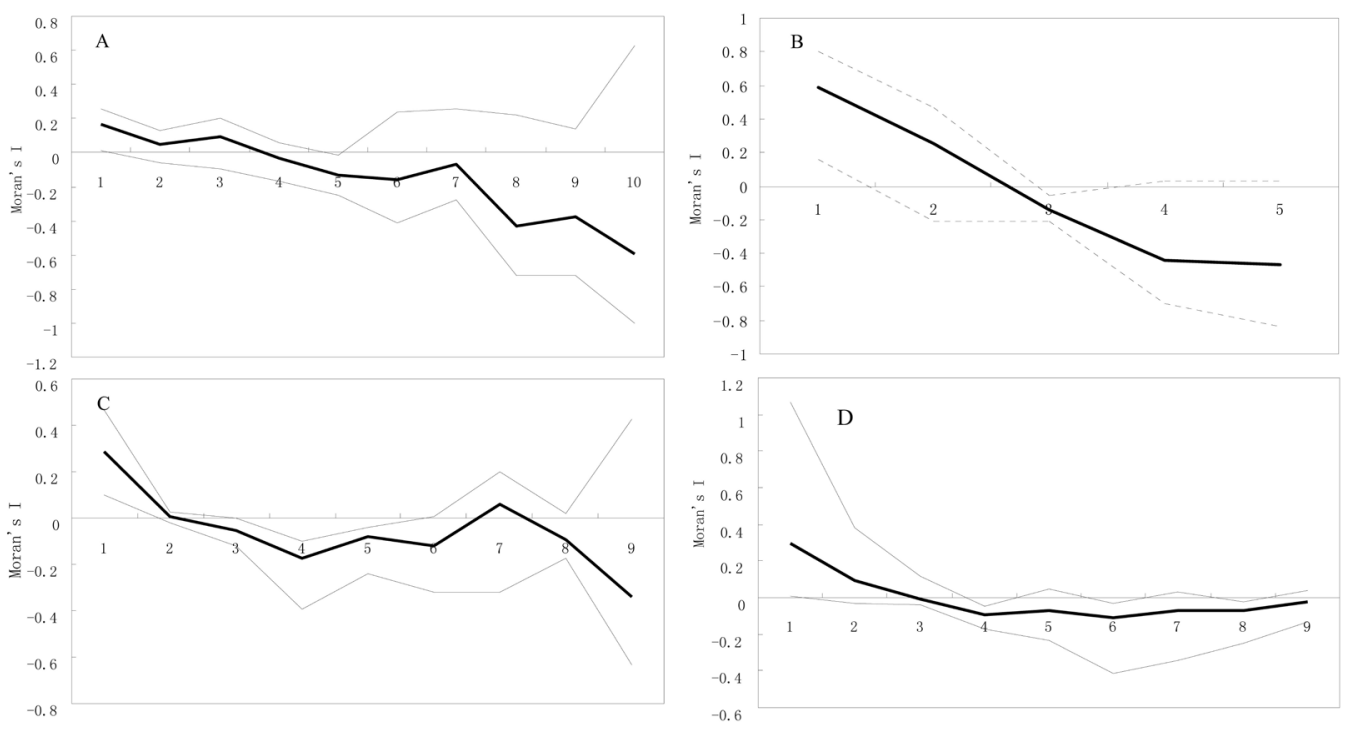

Figure 2. Correlograms of autocorrelation statistics in each distance class (m) of Caldesia grandis (equal interval correlograms). A. Patch BH-1 of the Bei Hai (BH) population; B. Patch BH-2 of the BH population; C. Patch GH-1 of the Guai Hu population; D. Patch LPH-1 of Lang Pan Hu population. 
For patch GH-1, seven loci with frequencies ranging from 20 to $80 \%$ were selected, and the average distance between individuals was $1 \mathrm{~m}$. Among the 70 calculated Moran's $I$ values, $21(30 \%)$ were statistically significant at the 5\% level (Table 4). At the first two of the 10 distance classes, more than $50 \%$ of the Moran's $I$ values were statistically significant. Within a short distance $(1 \mathrm{~m}), 100 \%$ of the statistically significant Moran's $I$ values were positive. With the distance increased, the positive Moran's $I$ values decreased and the negative values increased. Within the distance range of 9-10 m, 70\% of the statistical significant Moran's $I$ values were negative (see Table 4; Figure 2C).

Table 4. Spatial autocorrelation coefficients (Moran's $I$ ) for 10 distance classes in patch GH-1 in Guai Hu population of Caldesia grandis (equal interval correlograms).

\begin{tabular}{|c|c|c|c|c|c|c|c|c|c|c|c|}
\hline \multirow[t]{3}{*}{ Locus } & \multicolumn{10}{|c|}{ Distance class $(\mathrm{m})$ and No. of individual pairs } & \multirow[t]{3}{*}{ Cumulative probability } \\
\hline & 1 & 2 & 3 & 4 & 5 & 6 & 7 & 8 & 9 & 10 & \\
\hline & 104 & 126 & 91 & 61 & 57 & 62 & 77 & 63 & 42 & 20 & \\
\hline 1 & $0.21 * *$ & -0.02 & -0.12 & -0.14 & -0.08 & 0.01 & $0.20 *$ & -0.11 & $-0.40 * *$ & -0.28 & 0.039 \\
\hline 2 & $0.36 * *$ & 0.03 & -0.08 & $-0.39 * *$ & $-0.24 *$ & $-0.32 * *$ & $-0.32 * *$ & 0.02 & $0.43 * *$ & $0.46^{* *}$ & 0.000 \\
\hline 3 & $0.20 * *$ & 0.02 & 0.00 & -0.13 & -0.04 & -0.11 & 0.11 & -0.17 & $-0.40^{* *}$ & -0.30 & 0.034 \\
\hline 4 & $0.20 * *$ & 0.02 & 0.00 & -0.13 & -0.04 & -0.11 & 0.11 & -0.17 & $-0.40 * *$ & -0.30 & 0.034 \\
\hline 5 & 0.10 & 0.02 & -0.02 & -0.10 & -0.05 & -0.06 & 0.08 & -0.06 & $-0.32 *$ & -0.33 & 0.183 \\
\hline 6 & $0.47 * *$ & $\begin{array}{l}-0.02 \\
-0.01\end{array}$ & -0.06 & -0.14 & -0.06 & -0.11 & 0.12 & -0.08 & $-0.63 * *$ & $-1.00 * *$ & 0.000 \\
\hline 7 & $0.47 * *$ & -0.01 & -0.06 & -0.14 & -0.06 & -0.11 & 0.12 & -0.08 & $-0.63 * *$ & $-1.00 * *$ & 0.000 \\
\hline Average & 0.29 & 0.01 & -0.05 & -0.17 & -0.08 & -0.12 & 0.06 & -0.09 & -0.34 & -0.39 & \\
\hline Maximum & 0.47 & 0.03 & 0.00 & -0.10 & -0.04 & 0.01 & 0.20 & 0.02 & 0.43 & 0.46 & \\
\hline Minimum & 0.10 & -0.02 & -0.12 & -0.39 & -0.24 & -0.32 & -0.32 & -0.17 & -0.63 & -1.00 & \\
\hline
\end{tabular}

For patch LPH-1, a total of 10 loci with frequencies ranging from 20 to $80 \%$ were selected, and the average distance between individuals was $1 \mathrm{~m}$. Among the 100 calculated Moran's $I$ values, $21(21 \%)$ were statistically significant at the $5 \%$ level (Table 5). Within a short distance $(3 \mathrm{~m}), 100 \%$ of the statistical significant Moran's $I$ values were positive. With the distance increased, the positive Moran's $I$ values decreased and the negative values increased. Within the distance range of 4-8 m, 100\% of the statistical significant Moran's $I$ values were negative (see Table 5; Figure 2D).

Table 5. Spatial autocorrelation coefficients (Moran's $I$ ) for 10 distance classes in patch LPH-1 in Lang Pan Hu population of Caldesia. grandis (equal interval correlograms).

\begin{tabular}{|c|c|c|c|c|c|c|c|c|c|c|c|}
\hline \multirow[t]{3}{*}{ Locus } & \multicolumn{10}{|c|}{ Distance class $(\mathrm{m})$ and No. of individual pairs } & \multirow[t]{3}{*}{ Cumulative probability } \\
\hline & 1 & 2 & 3 & 4 & 5 & 6 & 7 & 8 & 9 & 10 & \\
\hline & 147 & 311 & 346 & 311 & 280 & 260 & 201 & 105 & 46 & 9 & \\
\hline 1 & $0.79 * *$ & $0.38 * *$ & $0.12 * *_{-}$ & $0.14 * *$ & $-0.21 * *$ & $-0.41 * *$ & $-0.34 * *$ & $-0.25 * *$ & -0.13 & 0.32 & 0.000 \\
\hline 2 & 0.01 & -0.03 & -0.03 & -0.06 & 0.05 & -0.03 & 0.03 & -0.02 & -0.02 & -0.39 & 1.000 \\
\hline 3 & 0.03 & -0.01 & -0.03 & -0.06 & 0.01 & -0.03 & 0.03 & -0.02 & 0.00 & -0.38 & 1.000 \\
\hline 4 & 0.03 & -0.01 & -0.03 & -0.06 & 0.01 & -0.03 & 0.03 & -0.02 & 0.00 & -0.38 & 1.000 \\
\hline 5 & $1.07 * *$ & $0.28^{* *}$ & -0.00 & $-0.17 * *$ & $-0.23 * *$ & $-0.34 * *$ & $-0.28 * *$ & $-0.18^{*}$ & 0.03 & 0.28 & 0.000 \\
\hline 6 & $0.72 * *$ & $0.12 * *$ & -0.04 & $-0.14 * *$ & $-0.11^{*}$ & $-0.17^{* *}$ & $-0.17 * *$ & -0.10 & 0.04 & 0.16 & 0.000 \\
\hline 7 & 0.07 & 0.04 & -0.02 & -0.05 & -0.05 & -0.03 & -0.01 & -0.03 & -0.03 & -0.04 & 1.000 \\
\hline 8 & 0.07 & 0.04 & -0.02 & -0.05 & -0.05 & -0.03 & -0.01 & -0.03 & -0.03 & -0.04 & 1.000 \\
\hline 9 & 0.07 & 0.04 & -0.02 & -0.05 & -0.05 & -0.03 & -0.01 & -0.03 & -0.03 & -0.04 & 1.000 \\
\hline 10 & 0.07 & 0.04 & -0.02 & -0.05 & -0.05 & -0.03 & -0.01 & -0.03 & -0.03 & -0.04 & 1.000 \\
\hline Average & 0.30 & 0.09 & -0.01 & -0.09 & -0.07 & -0.11 & -0.07 & -0.07 & -0.02 & -0.05 & \\
\hline Maximum & 1.07 & 0.38 & 0.12 & -0.05 & 0.05 & -0.03 & 0.03 & -0.02 & 0.04 & 0.32 & \\
\hline Minimum & 0.01 & -0.03 & -0.04 & -0.17 & -0.23 & -0.41 & -0.34 & -0.25 & -0.13 & -0.39 & \\
\hline
\end{tabular}




\section{DISCUSSION}

Spatial structuring, or nonrandomness, can be strongly influenced by many aspects of population genetics (Sokal, 1979; Epperson, 1990). Isolation by distance, limited gene flow, or seed dispersal should cause neighboring individuals in plant populations to be genetically related, and the spatial autocorrelation between individuals should decrease with increasing distances (Wright, 1946; Epperson, 1990; Li et al., 2002). In addition, local environments may also partly explain significant genetic clustering (Hamrick and Allard, 1972; Allard et al., 1977; Epperson, 1993; He et al, 1999). The spatial autocorrelation analysis in this study showed that within short distances, the RAPD genetic variations were not randomly distributed in patches BH-1 (3 m), BH-2 (2 m), and GH-1 (1 m), indicating that individuals within the range of 1-3 $\mathrm{m}$ were similar; as distance increased, the negative Moran's $I$ values increased in each of these patches, indicating that the individuals within a larger distance range were randomly distributed. For patch LPH-1, the vast majority of RAPD loci were randomly distributed; only a few loci displayed a patchy distribution model, indicating that no clear spatial genetic structure existed. The different spatial genetic variation patterns revealed in the patches of $C$. grandis populations may be attributed to environmental differences.

C. grandis can produce many flowers from early July to late September, with a peak in August. The effective pollinators are bees (Insecta; Hymenoptera; Gituru et al., 2002). This species is a primarily outcrossing species (Chen et al., 2007). Although sexual reproduction may have played an important role in generating genetic variation in the history of the plant, seedling recruitment is now rarely observed (Gituru et al., 2002; Chen et al., 2006). Currently, C. grandis depends mainly on asexual reproduction through inflorescence bulbils. The ramets are mainly distributed around their maternal plants, which may contribute to the patchy distribution pattern of RAPD variation in the $\mathrm{BH}$ and $\mathrm{GH}$ populations. The $\mathrm{BH}$ population of $C$. grandis displayed a patchy or scattered distribution in a "floating blanket" type swamp. When the inflorescence bulbils of $C$. grandis mature and drop off, they mainly remain around the maternal plants because little water flow or other environmental factors are present to spread the bulbils farther. Thus, the populations displayed significant patchy distribution of RAPD genetic variation.

In patch GH-1, although the GH marsh has a higher water level and C. grandis lives in deeper water, little fluctuation in water level occurs. Moreover, the dominant plant species in the GH marsh is B. schreberi, and the floating leaves of B. schreberi cover most of the water surface, which presents an obstacle to the spread of the inflorescence bulbils of $C$. grandis. This environmental characteristic may have resulted in the similarities in RAPD genetic variation (patchy distribution) in the GH and $\mathrm{BH}$ populations. However, the RAPD variation in patch LPH-1 exhibited a different pattern (random distribution) from that in the $\mathrm{GH}$ and $\mathrm{BH}$ populations. Although C. grandis individuals in the LPH population showed patchy distribution, a sluggish stream flows across the population, and the water flow can carry the inflorescence bulbils of $C$. grandis far from the maternal plants, which may cause neighboring individuals in this population to be genetically dissimilar. The differing local habitat of the LPH population of $C$. grandis may have led to a RAPD spatial distribution that varied from that in the other populations.

The insights gained from exploring the spatial genetic structure of populations of rare and endangered species are important for developing a sound strategy for protecting them. The spatial patterns of genetic variation found in $C$. grandis populations provide important baseline data for the conservation of this species, especially for sampling strategies in ex situ con- 
servation. For example, the genetic variation in the $\mathrm{BH}$ and $\mathrm{GH}$ populations was distributed in a significant patchy pattern, which requires us to sample at not less than $3 \mathrm{~m}$ to avoid repeated sampling of genetically similar individuals. Owing to the less patchy genetic structure in the LPH population, however, sampling should not be limited to the same distance.

\section{ACKNOWLEDGMENTS}

We thank Jing-Yuan Wang and Fan Liu for their help with fieldwork, and Xia Jing and Zhi-Yuan Du for their assistance in the laboratory. Research supported by grants from the One Hundred Person Project of the Chinese Academy of Sciences granted to WQF (KSCX2-YWZ-0805) and from the National Natural Science Foundation of China (\#30970195 and \#30970202).

\section{REFERENCES}

Allard RW, Kahler AL and Clegg MT (1977). Measuring Selection in Natural Populations. Springer-Verlag, NewYork.

Berg EE and Hamrick JL (1994). Spatial and genetic structure of two sandhills oaks: Quercus laevis and Quercus margaretta (Fagaceae). Am. J. Bot. 81: 7-14.

Born C, Hardy OJ, Chevallier MH, Ossari S, et al. (2008). Small-scale spatial genetic structure in the Central African rainforest tree species Aucoumea klaineana: a stepwise approach to infer the impact of limited gene dispersal, population history and habitat fragmentation. Mol. Ecol. 17: 2041-2050.

Bouza N, Caujape-Castells J, Gonzalez-Perez MA, Batista F, et al. (2002). Population structure and genetic diversity of two endangered endemic species of the Canarian laurel forest: Dorycnium spectabile (Fabaceae) and Isoplexis chalcantha (Scrophulariaceae). Inter. J. Plant Sci. 163: 619-630.

Bucci G and Menozzi P (1995). Genetic-variation of RAPD markers in a Picea-abies Karst - Population. Heredity 75: 188-197.

Chen JM, Gituru WR, Wang YH and Wang QF (2006). The extent of clonality and genetic diversity in the rare Caldesia grandis (Alismataceae): comparative results for RAPD and ISSR markers. Aquat. Bot. 84: 301-307.

Chung MG and Epperson BK (1999). Spatial genetic structure of clonal and sexual reproduction in populations of Adenophora grandiflora (Campanulaceae). Evolution 53: 1068-1078.

Cliff AD and Ord JK (1981). Spatial Processes: Models and Applications. Pion, London.

Cook CDK (1996). Aquatic and Wetland Plants of India: A Reference Book and Identification Manual for the Vascular Plants Found in Permanent or Seasonal Fresh Water in the Subcontinent of India South of the Himalaya. Oxford University Press, Oxford.

Degen B, Caron H, Bandou E, Maggia L, et al. (2001). Fine-scale spatial genetic structure of eight tropical tree species as analysed by RAPDs. Heredity 87: 497-507.

Epperson BK (1990). Spatial Patterns of Genetic Variation Within Plant Populations. In: Plant Population Genetics, Breeding, and Genetic Resources (Brown AHD, Clegg MT, Kahler AL and Weir BS, eds.). Sinauer Associate Inc., Sunderland, 229-253.

Epperson BK (1993). Spatial and space-time correlations in systems of subpopulations with genetic drift and migration. Genetics 133: 711-727.

Epperson BK and Clegg MT (1986). Spatial-autocorrelation analysis of flower color polymorphisms within substructured populations of morning glory (Ipomoea purpurea). Am. Nat. 128: 840-858.

Escudero A, Iriondo JM and Torres ME (2003). Spatial analysis of genetic diversity as a tool for plant conservation. Biol. Conser 113: 351-365.

Fritsch P and Rieeseberg LH (1992). High outcrossing rates maintain male and hermaphrodite individuals in populations of the flowering plant Datisca glomerata. Nature 359: 633-636.

Gituru WR, Wang QF, Wang Y and Guo YH (2002). Pollination ecology, breeding system, and conservation of Caldesia grandis (Alismataceae), an endangered marsh plant in China. Bot. Bull. Acad. Sin. 43: 231-240.

Hamrick JL and Allard RW (1972). Microgeographical Variation in Allozyme Frequencies in Avena barbata. Proc. Natl. Acad. Sci. U. S. A. 69: 2100-2104.

He TH, Yang J and Rao GY (1999). Spatial autocorrelation analysis of plant population genetic variation. Chin. Bull. Bot. 16: 636-641 
He TH, Rao GY, You RL, Ge S, et al. (2000). Spatial autocorrelation of genetic variation in three stands of Ophiopogon xylorrhizus (Liliaceae s.1.). Ann. Bot. 86: 113-121.

Hedrick PW (1986). Genetic polymorphism in heterogeneous environments: a decade later. Ann. Rev. Ecol. Syst. 17: 535-566.

Heun M, Murphy JP and Phillips TD (1994). A comparison of RAPD and isozyme analysis for determining the genetic relationships among Avena sterilis L. accessions. Theor. Appl. Genet. 87: 689-696.

Heywood JS (1991). Spatial analysis of genetic variation in plant populations. Ann. Rev. Ecol. Syst. 22: 335-355.

Huff DR, Peakall R and Smouse PE (1993). RAPD variation within and among natural populations of outcrossing buffalograss [Buchloe dactyloides (Nutt.) Engelm.]. (Theor. Appl. Genet. 86: 927-934.

Li A, Luo YB and Ge S (2002). Spatial autocorrelation study of population genetic structure of two orchid species. Biodiversity 10: 249-257.

Murawski DA and Hamrick JL (1990). Local genetic and clonal structure in the tropical terrestrial bromeliad, Aechmea magdalenae. Am. J. Bot. 77: 1201-1208.

Reisch C, Schurm S and Poschlod P (2007). Spatial genetic structure and clonal diversity in an alpine population of Salix herbacea (Salicaceae). Ann. Bot. 99: 647-651.

Sokal RR (1979). Ecological Parameters Inferred from Spatial Correlograms. In: Contemporary Quantitative Ecology and Related Ecometrics (Patil GP and Rosenzweig ML, eds.). International Cooperative Publishing House, Fairland, 167-196.

Sokal RR and Oden NL (1978a). Spatial autocorrelation in biology 1. Methodology. Biol. J. Linn. Soci. 10: 199-228.

Sokal RR and Oden NL (1978b). Spatial autocorrelation in biology 2. Some biological implications and four applications of evolutionary and ecological interest. Biol. J. Linn. Soci. 10: 229-249.

Sokal RR, Jacquez GM and Wooten MC (1989). Spatial autocorrelation analysis of migration and selection. Genetics 121: 845-855.

Tani N, Tomaru N, Tsumura Y, Araki M, et al. (1998). Genetic structure within a Japanese stone pine (Pinus pumila Regel) population on Mt. Aino-Dake in central Honshu, Japan. J. Plant Res. 111: 7-15.

Torres E, Iriondo JM, Escudero A and Perez C (2003). Analysis of within-population spatial genetic structure in Antirrhinum microphyllum (Scrophulariaceae). Am. J. Bot. 90: 1688-1695.

Vornam B, Decarli N and Gailing O (2004). Spatial distribution of genetic variation in a natural beech stand (Fagus sylvatica L.) based on microsatellite markers. Conser. Genet. 5: 561-570.

Warternberg D (1989). SAAP 4.3.: A Spatial Autocorrelation Analysis Program. Exeter Software, Setauket.

Wright S (1946). Isolation by distance under diverse systems of mating. Genetics 31: 39-59. 